\title{
Evidence-Based Practice Utilization and Associated Factors Among Nurses in Public Hospitals, Addis Ababa, Ethiopia
}

Kidist Assefa ( $\sim$ Kidiassefa13@gmail.com )

Kotebe Metropolitan University

Zewdu Shewangizaw

Kotebe Metropolitan University

\section{Research Article}

Keywords: Hospitals, Evidence-based practice, Utilization, Nurses.

Posted Date: May 13th, 2021

DOl: https://doi.org/10.21203/rs.3.rs-477800/v1

License: (c) (1) This work is licensed under a Creative Commons Attribution 4.0 International License.

Read Full License 


\section{Abstract}

Background: To make an evidence-based practice, clinical research and updates matters, thus the result of this study will help nurses to exercise evidence-based practice for the utmost care to their patients. With regards all the various programs and strategies to promote the use of clinical research and updates, there is still a gap in practicing. A number of studies from various countries have reported that nurses' experience of evidence-based practice is low. In Ethiopia, there is a gap on evidence-based nursing practice.

Objective: To assess evidence-based practice utilization and associated factors among nurses at public hospital Addis Ababa, Ethiopia, 2020.

Methods: A mixed-method approach (concurrent triangulation) was employed from November to December, 2020 among 422 study participant. Semi-structured and pretested self-administered questioners were used to collect data. Then the collected data were cleaned, coded, and entered into Epi data version 4.6 and exported to SPSS version 22 for analysis. The result was displayed through simple and multiple linear regressions. The total scores were expressed using regression coefficient ( $\beta$ ), $95 \%$ Confidence interval. For the qualitative study, in-depth interviews were conducted with a purposive sampling method. The data were entered and analyzed using open code software version 4.0.

Results: Of 422 distributed questionnaires, all were completed with $100 \%$ response rate. EBP exercised by the participant to the degree of $28.4 \%, 48.8 \%$ and $8.8 \%$ respectively to low, medium and high level. Those with a good knowledge about EBP, increases their implementation by 0.338 . Educational status of nurses was significantly associated with evidence-based practice utilization $(\beta=0.647, P=0.02)$. Having a managerial support will increase the implementation by 0.835 .

Conclusion: Small number of participants had implemented EBP to a high level. Evidence-based nursing practice was positively associated with knowledge, attitude, and educational status, lack of managers' support and availability of information resources. Intervention programs on awareness creation, training, resource provision, and curriculum issues to improve implementation of evidence based nursing practice by stakeholders are recommended.

\section{Introduction}

Evidence-Based Practice (EBP) is a use of evidence in clinical decision-making by integrating clinical experience and incorporating patient values and preferences, in the practice of professional patient care (1). It is about making decisions by integrating individual clinical experts with the best available external clinical evidence from systematic research $(2,3)$. The accessibility of information and the development of science has led to considerable improvements in health outcomes all over the world. However, differences in outcomes, health inequalities, and weakly performing health services continue to present a real challenge to all nurses and this results in EBP to be acknowledged internationally(4). 
Evidence-based nursing implements the most up to date methods of providing care, which has been proven through the appraisal of high-quality studies and statistically significant research findings. Evidence-based nursing practice is a process founded on the collection, interpretation, appraisal, and integration of valid, clinically significant, and applicable research $(5,6)$.

The EBP process need not be onerous and includes five elements: formulating an appropriate question, performing an efficient literature search and performing research, critically appraise the best available evidence, applying the best evidence to clinical practice assessing outcomes of care, and sharing outcomes(7).

The EBP model has been accepted by healthcare professionals as an important means to improve the quality of patient care, but its implementation is still deficient (8). Poor utilization of evidence-based practice has an impact on clinical outcomes, patient retention, and reimbursement clams (9). Poorly informed decision-making is one of the main reasons that fails the service to be delivered optimally and can also contribute variation in practice which makes it less efficient, ineffective, and inequitable. Half of the world's death could be prevented with simple cost-effective interventions (10-12). Despite this many countries worldwide have advocated the importance of evidence-based practice among nurses but its utilization was not satisfactory (13). Hence study in India showed that only $45.8 \%$ of nurses in the hospital had implemented EBP (14).

Studies in Africa showed that utilizing EBP is challenging due to Africa fall behind research and lack of funds. But, some African countries like, South Africa, Botswana, Nigeria, Kenya Malawi, and Egypt are advocating evidence-based practice $(1,8,9)$. A study conducted in TASH among nurses revealed that $57.6 \%$ of them utilized EBP (11). A similar study in Jimma also revealed that $51.8 \%$ of nurses utilize EBP(15). Factors like sociodemographic status, organizational factors (administrative support ), and different individual factors ( knowledge and skill to evaluate research findings) often fomented nurses' utilization of $\operatorname{EBP}(11,16)$.

Even though several systematic reviews and resources for evidence-based practice had risen, the application of evidence-based practice remains challenging for nurses $(13,17,18)$. In Ethiopia, the federal ministry of health reported that as there is a lack of skilled nurses who could help to synthesize evidence for policy-making (19). So, this study seeks to explore the level of EBP implementation and will give information on concerning factors, which hinder the EBP among nurses working under Addis Ababa public hospitals.

\section{Methodology}

\section{Study setting and design}

Mixed method approach was employed from November, 2-December, 2, 2020. The study was conducted in Addis Ababa public health hospitals which contain 7496 nurses. There are 13 governmental hospitals in Addis Ababa, of which five hospitals were selected by simple random sampling. 


\section{Study populations}

Four hundred and twenty two nurses who were working in the selected public hospitals.

\section{Sample size and sampling procedures}

The sample size was determined based on the single population proportion formula and an assumption of $51.8 \%$ was taken from a study conducted in Jimma hospital .Assuming a $10 \%$ non-response rate, a total sample size of 422 nurses were needed. Overall sample was proportionally allocated to each hospital based on the number of nurses and simple random sampling was used to select participants of the study. Those nurses who were having work experience more than 6 months were included and nurses who were on annual and maternity leave were excluded.

\section{Data collection tools}

Quantitative data was collected by using a semi-structured self-administered questionnaire adapted from previous literatures $(11,15,20,21)$. The quantitative study tools contain five parts with 41 items.

Qualitative data involved interviews with key informants selected to explore their perspectives. Interview guides were used to get information concerning utilization of EBP and associated factors from the nurse's point of view. Detail information was explored with complete picture of utilization of EBP and associated factors. Eight open ended questions were prepared with their probes.

\section{Data collection procedures}

Quantitative data were collected from nurses by two data collectors through distributing structured selfadministered questioner for the nurses after explaining the purpose and technique of filling the questionnaire. Continuous follow-up and supervision was made by one facilitator and Principal Investigator (PI) throughout the data collection period. Qualitative data was collected by PI from key informants. Key informants took approximately for in-depth interview based on purposive sampling. Each in depth interview was taken 30-35 minutes. Notes and audio recorder were used for recording the information obtained from key informants.

\section{Data quality control assurance}

Quantitative data was checked daily for completeness and consistency throughout the data collection period by facilitators and $\mathrm{Pl}$, then each completed questionnaire was given a unique code. Prior to data collection pre-test was conducted on $5 \%$ of the total sample size in order to check the reliability of the instrument, to estimate the time needed to collect data and to modify the questionnaire accordingly. The tool was checked for reliability (internal consistency) using the Cronbach's alpha coefficient which was 0.9. One day training was provided for facilitators by the PI. For qualitative data the individual interviewee was interviewed separately to prevent the contamination of information.

\section{Study variables}


Dependent variables: Evidence based practice utilization

In this study the scale that measured implementation of EBP includes seven items with minimum score of 7 and maximum score of 35 and treated as continues variable. Moreover, the score on implementation of evidence based nursing practice (EBNP) was transformed into Tertian classification for descriptive purpose (20). The higher the score the higher the implementation of EBNP. The independent variables include: socio-demographic factors (age, sex, marital status, educational status, working unit), organizational factors (administrative support, colleague support, access to different resources) and characteristic of individual (knowledge, attitude, position in the health facility, and years of experience).

\section{Statistical analysis}

The data were carefully entered into Epidata V4.6, edited and cleaned for inconsistencies and missing values. Data was analyzed using SPSS version 22. Descriptive statistic was used to summarize data. Bivariate and multivariable linear regression was used to assess the association between dependent variable and independent variables. The variable with $p$-value less than 0.05 was taken as having significant association.

\section{Ethical considerations}

Ethical clearance was obtained from Addis Ababa public health research and emergency management directorate and Ethical Committee of Kotebe Metropolitan University Menelik II Medical and Health Science College. Then the data collectors were distributed permission letters for all selected study hospitals. A formal letter was also submitted to all concerned bodies to obtain their co-operation. Data collection was conducted after verbal informed consent obtained from participants, who were taken from each selected participant after explanation of the survey purpose, description of the benefits, and an offer to answer all inquiries to the respondents.

\section{Results}

\section{Socioeconomic and demographic characteristics}

Among 422 respondents, $58.5 \%$ were female. The mean age was 32 years ( $S D \pm 7.77)$, minimum age is 25 and maximum age is 59 years old. Majority were BSc holders. Staff nurses constituted the majority of our sample which is 389 (92.2\%). The majority of participants 82 (19.4\%) worked on emergency department (Table 1).

Table 1: Sociodemographic characteristics of Nurses at Public Hospitals, Addis Ababa, Ethiopia, $2020(n=422)$. 


\begin{tabular}{|lcc|}
\hline Variables & Frequency & Percentage \\
\hline Sex & & \\
\hline Male & 175 & 41.5 \\
\hline Female & 247 & 58.5 \\
\hline Marital status & & \\
\hline Single & 197 & 46.7 \\
\hline Ever married & 225 & 53.3 \\
\hline Educational level & & \\
\hline Diploma & 18 & 4.3 \\
\hline BSC and above & 404 & 95.7 \\
\hline Working position & & \\
\hline Head & 33 & 7.8 \\
\hline Staff & 389 & 92.2 \\
\hline
\end{tabular}

\section{Level of evidence-based practice}

A total 422 participants involved in different level of evidence-based practice activities. Out of 422 participant $363(86 \%)$ had involved in different EBP activities to different level (from seldom to often). Total score was computed for individuals EBP and Tertian classification was done on the total score of EBP activity measurements for descriptive purpose. Accordingly, about 120 (28.4) of the respondents implemented EBP to low level (sometimes), about 206(48.8\%) of the respondents implemented EBP to medium level and about 37 (8.8\%) of respondents implemented EBP to high level (Table 2).

Table 2: Distribution of respondents by involvement in EBP activities at Public Hospitals, Addis Ababa, Ethiopia, 2020 


\begin{tabular}{|llllllllllll|}
\hline \multirow{2}{*}{ Activities } & \multicolumn{3}{l}{ Never } & \multicolumn{3}{c}{ Seldom } & \multicolumn{3}{c|}{ Occasionally } & \multicolumn{3}{c|}{ Regularly } & \multicolumn{2}{c|}{ Often } \\
\cline { 2 - 13 } & $\mathrm{N}$ & $\%$ & $\mathrm{~N}$ & $\%$ & $\mathrm{~N}$ & $\%$ & $\mathrm{~N}$ & $\%$ & $\mathrm{~N}$ & $\%$ \\
\hline Formulate question & 119 & 28.2 & 139 & 32.9 & 119 & 28.2 & 36 & 8.5 & 9 & 2.1 \\
\hline $\begin{array}{l}\text { Search for research findings } \\
\text { or evidence }\end{array}$ & 105 & 24.9 & 128 & 30.3 & 138 & 32.7 & 43 & 10.2 & 8 & 1.9 \\
\hline Read for research findings & 87 & 20.6 & 111 & 26.3 & 157 & 37.2 & 55 & 13 & 12 & 2.8 \\
\hline Critically appraised evidence & 117 & 27.7 & 99 & 23.5 & 147 & 34.8 & 51 & 12.1 & 8 & 1.9 \\
\hline $\begin{array}{l}\text { Apply best evidence to } \\
\text { clinical practice }\end{array}$ & 99 & 23.5 & 112 & 26.5 & 136 & 32.2 & 60 & 14.2 & 15 & 3.6 \\
\hline $\begin{array}{l}\text { Assess or evaluate } \\
\text { outcomes of care }\end{array}$ & 95 & 22.5 & 116 & 27.5 & $1 ! 4$ & 27 & 84 & 19.9 & 13 & 3.1 \\
\hline Teaching new evidence & 106 & 25.1 & 111 & 26.3 & 122 & 28.9 & 65 & 15.4 & 18 & 4.3 \\
\hline
\end{tabular}

From qualitative study key informants mentioned about utilization of EBP that they have never heard about EBP but they explained as it helps for having a better care for the patient. A 28 years old matron from Rasdesta Dametew hospital described that they had no information regarding the utilization of EBP but they know that it helps for having a good patient care.

"We have no information about utilization of EBP in clinical practice and also utilization of EBP in clinical practice is good for patient's care improvement, it saves time and increases their satisfaction." [Participant 7 age 28, sex male, BSc. Nurse, Matron]

Another 36 year-old matron from Minilik explained that majority of nurses explained that they used different sources to have a recent and updated evidence for their clinical practice but not applied frequently

"Most of nurses used evidence from hospital protocols, guidelines and others asked information from senior staff nurses /colleagues and other health professionals like doctors and most of nurses sometimes used EBP." [Participant 1 age 36, sex male, BSc. Nurse, Matron]

\section{Factors associated with utilization of EBP}

\section{Knowledge about evidence-based practice}

Among total respondents, 187 (44.3\%) were categorized as knowledgeable about EBP as those nurses who score $>=$ to the calculated demarcation threshold formula.

\section{Attitudes about EBP}


Among total respondents, 366(86.7\%) were categorized as having favorable attitude about EBP as they score $>=$ mean score and from those nurses who had a favorable attitude.

\section{Organizational and individual factors}

Participants were asked about the organizational, and personal provider factors that posed barriers to incorporate EBP into their clinical practice. The most frequently agreed barriers were an authority to change the practice (66.2\%), lack of manager's support and incentive (61.3\%), culture of the team to change the practice (64.5\%), and physician cooperation with implementation of new evidence $(61.7 \%)$. Almost more than half of respondents $(60 \%)$ said that the available national treatment guidelines and protocols were difficult to understand.

From qualitative results majority of key informants mentioned that there are different barriers to utilize of EBP such as: insufficient resources (e.g. current literature; internet access; updated guidelines; computers); lack of financial support (incentives); closed minds (we have always done it this way); lack of library and lack of support (e.g. management, physicians),lack of time due to workload, nurses' willingness to perform new procedure to patient care, nurse's ability in finding new procedure, nurses communication skill with nurses and physicians.

A 38 years old case team leader from Minilik hospital explained that they face many challenges to utilize EBP.

"......there are experienced nurses/ colleague, doctors and other health professionals to support nurses in their clinical practice but they have many challenges from their colleagues......"' [participant 3 age 38, sex male, Nurse, Case team leader]

"nurses have no interest, they don't want to read and update themselves even they have no confidence and majorly no hospital library, updated guidelines, internet services, motivation, enough training and no enough computers for nurses to updating themselves "[participant 6 age 35, sex male, Medical doctor, Medical director]

The 30 years old case team leader from St.Pawlos hospital described that they have a crowded working environment which makes it harder to utilize EBP.

"There are times when I do not know things and I do not have even time to sit down and also most of nurses were ask doctors and colleagues during ward rounds while some most of them have no interest in updating themselves."[Participant 3 age 30, sex male, BSc. Nurse, case team leader]

However, some of the key informants described as there is some movement regarding giving training for staffs but still there is a gap in having a good training session on how to implement EBP. 
"Even if it is not enough sometimes there is training for some nurses, hospital protocols and guidelines but most of them doesn't apply on their practice."[Participant 4 age 35, sex female, BSc. Nurse, case team leader]

\section{Factors associated with implementation of evidence based nursing practice}

Knowledge about EBP was positively associated with EBNP implementation ( $\beta=0.338, P=0.000)$. Those with a good knowledge about EBP, increases their implementation by 0.338 . Educational status of nurses was significantly associated with evidence-based practice implementation $(\beta=0.647, P=0.02)$. Having a BSc and above increases evidence-based practice by 0.647 . Nurses attitude regarding EBP was positively associated with $\operatorname{EBNP}(\beta=0.139, P=0.018)$. Nurses with a favorable attitude regarding EBNP increases evidence based practice by 0.139 .

Managers support and incentive was significantly associated with nurses evidence-based practice $(\beta=0.103, P=0.018)$. Having a managerial support will increase the implantation by 0.103 . Insufficient information to find new guidelines or protocols was significantly associated with implementing evidencebased practice $(\beta=1.196, P=0.000)$.

\section{Discussion}

This study aimed to determine the level of EBP and factors associated with EBP. In this study, EBP among nurses of the public hospital in Addis Ababa was found to be $86 \%$.

In this study, respondents were participated in each activity of EBP with different levels of involvement, but for its simplicity the score was summed up and transformed into Tertian classification. Accordingly, about 120 (28.4\%) of the respondents had implemented EBP to low level (sometimes), about 206 (34.1\%) of the respondents had implemented EBP to medium level (usually) and $37(8.8 \%$ ) of the respondents implemented EBP to high level (always). This finding was consistent with the study done in Sweden by Bostrom et al, 2013 in which (60\%), approximately similar level of EBP implementation was reported (19\% asked clinical questions and performed searches in data bases, $56 \%$ used information sources, $31 \%$ appraised the literature, $30 \%$ participated in practice development, and $34 \%$ participated in evaluating clinical practice to high extent)(22). Also finding from this study was analogous with the study conducted in South Africa, in which 35.6, 32.9, and 31.5\% use EBP frequently, moderately and rarely, respectively. Even though in this study small numbers of respondents were frequently involved in EBP about (18.4\%), the finding is similar with finding from South Africa (23). This study was also analogous with study conducted in Jimma among nurses, in which $81 \%$ of respondents applied EBP(20). On the other hand the finding from this study is higher than the study done in Tikur Anbsa hospital in Addis Ababa, in which 57.6\% participants applied EBP. Of them, 64 (52.8\%), 38 (31.4\%) and 19 (15.7\%) applied EBP sometime, usually and always respectively (11) .

This could be due to the sample size difference. In this study, the sample is somewhat higher than the study done in Tikur Anbsa hospital. The other possible justification may be due to the study was done in 
five different hospitals. Concerning perceived barriers to research utilization the most frequently agreed barriers were an authority to change the practice $(66.2 \%)$, lack of managers support and incentive $(61.3 \%)$, culture of the team to change the practice (64.5\%), and physician cooperation with implementation of new evidence (61.7\%).

This finding was similar with studies done in USA, organizational factor has been consistently reported as greatest perceived factor(21). On the other hand, when we compare top perceived barriers to research utilization with seven other studies except this study, most of the studies reported that insufficient time to read and implement new idea' was the most reported barrier $(5,24)$.

In this study, being knowledgeable about EBP was positively associated with EBP implementation $(\beta=0.338, P=0.000)$. This finding was similar in pattern with study done in USA where nurses who reported having greater knowledge of EBP also reported a greater extent of evidence-based care $(r=0.42, p$ $<0.0001)(21)$. Similarly, the study done in Iran is in line with this finding where nurses who knew the definition of evidence-based nursing were more likely to utilize research findings in clinical practice than those who did not know (Pearson $r 2=23.912, P=0.02)(24)$. Similarly, in our country, one study that was conducted in Tikur Anbsa hospital reported that those nurses who have knowledge were 3 times more likely to practice EBP than those who do not have knowledge (11). Another study conducted in Jimma among nurses reveled that nurses who reported having higher knowledge of EBP also reported a greater extent of evidence-based nurses who reported having greater knowledge of EBP also reported a greater extent of evidence-based practice $(\beta=0.76, P=0.008)(20)$. This implies that those nurses who have knowledge about EBP were more likely used EBP than those have no knowledge. This might be related with having up to dated information about EBP through media, training or searching internet.

Under this study, having a favorable attitude about EBP was positively associated with EBP implementation ( $\beta=0.139, P=0.018)$. This finding was similar with the study conducted in Amhara region governmental hospital where nurses attitude have a greater extent to utilize $\operatorname{EBP}(\mathrm{AOR}=5.02,95 \% \mathrm{Cl}=(1.2$, 21.5) (29).

Educational status of participants was positively associated with EBP implementation ( $\beta=0.647$, $P=0.02)$. This finding was similar with the study conducted in Jimma where nurses who have a greater educational status have a greater extent to utilize EBP $(A O R=3.186,95 \% \mathrm{Cl}=(1.634,6.210)(15)$

\section{Limitations}

Self-administered questionnaire was used to obtain the data which may introduce information biases and under or overestimate the result. Social desirability bias may affect the result of this study.

\section{Recommendation}

Based on the finding of this study the following recommendations has made. 
There has to be intervention program to facilitate the implementation of evidence in nursing practice by the respective stakeholders (Ethiopian Federal Ministry of Health, Addis Ababa Health Bureau, educators of nursing education and hospital administrations).

Resources necessary to implement evidence-based nursing practice should be provide by hospital administration with Ethiopian Federal Ministry of Health, Addis Ababa Health Bureau and other NGOs.

Intervention programs concerning organizational communication with issue on evidence-based practice implementation should be done together with nurses and physician to create supportive staff by hospital administration of Addis Ababa public hospitals.

Since there is still a gap of awareness on evidence based nursing practice curriculum planners should take into consideration to include the principle of evidence based nursing practice in Ethiopian nursing education programs especially in the undergraduate nursing education curriculum.

\title{
Conclusion
}

This study revealed that few number of nurses implement EBP to high level. In general individual and organizational factors were found to be the greatest obstacle to implement EBP. Evidence-based practice was associated with knowledge and attitude regarding EBP, educational status of nurses, lack manager's support and incentive and insufficient information to find new guidelines/protocols.

To conclude common EBP in public hospitals, continuous hard works are needed. Not only nurses need to become more familiar with EBP, but also other health professionals. Future research should therefore also focus on the assessment of EBP among all health professional.

\section{Abbreviations}

\author{
AA Addis Ababa
}

ALERT All Africa Leprosy Rehabilitation and Training Center

AOR Adjusted Odds Ratio

CSA Central Statistical Agency of Ethiopia

DC Data Collector

EBP Evidence-Based Practice

EOPD Emergency Outpatient Department

LMIC Low Middle Income Countries 
NHRS National Health Research System

PI Principal Investigator

SPSS Statistical Package for the Social Science

TASH Tikur Anbsa Specialty Hospital

UNESCO United Nation Educational, Scientific and Cultural Organization

WHO World Health Organization

\section{Declarations}

\section{Ethics approval and consent to participate}

Ethical clearance was obtained from Addis Ababa public health research and emergency management directorate and Ethical Committee of Kotebe Metropolitan University Menelik II Medical and Health Science College. Then the data collectors were distributed permission letters for all selected study hospitals. A formal letter was also submitted to all concerned bodies to obtain their co-operation. Data collection was conducted after verbal informed consent obtained from participants, who were taken from each selected participant after explanation of the survey purpose, description of the benefits, and an offer to answer all inquiries to the respondents.

All methods starting from the study area to the ethical consideration were carried out in accordance with relevant guidelines and regulations which were taken from John W. Creswell and J. David Creswell.

\section{Authors' contribution}

Kidist Assefa and Zewdu Shewangizaw conceive the study, were involved in the study design, review of the article, analysis, and report writing, and drafted the manuscript. All the authors and approve the final manuscript.

\section{Data availability}

Data set used or analyzed during the current study are available from the corresponding author on reasonable report.

\section{Consent for publication}

Not applicable.

\section{Disclosure}

To keep privacy of the respondents, the name and any identity were not included in the data collection. 


\section{Conflict of interest}

We declared that we have no conflict of interest.

\section{Funding}

It was self-covered and no funding from any agent.

\section{Acknowledgment}

First, our gratitude goes to Kotebe Metropolitan University Menelik II Medical and Health Science College for support in the accomplishment of this study. We would also like to thank all data collectors and respondents for their participation in the study.

\section{References}

1. Stevens KR. The impact of evidence-based practice in nursing and the next big ideas. OJIN: The Online Journal of Issues in Nursing. 2013;8(2). doi:10.3912/OJIN.

2. Rangachari P RP, Rethemeyer K. Awarness of evidence-based practices alone does not translate to implementation/ : insights from implementation research. Qual Mang Health Care. 2013.

3. United Nations Economic Commission for Africa AS, Technology and Innovation Review. African Science, Technology and Innovation Review . 2013.

4. Cummings GG OS, Biondo PD, Stiles CR, Yurtseven O, Fainsinger RL, et al. Effectiveness of Knowledge Translation Interventions to Improve Cancer Pain Management. J Pain Symptom Manage. 2011;41:915.

5. Kania-Lchance D.M. Best P.J.M MMR, and Ghosh A.K. Evidence based practice and the nurse practioioner. Nurse Pract. 2006;31(10):46-54. (Pub Med).

6. BM. M. Evidence based practice in nursing healthcare a guide to best practice Philadelphia PA. Lippincott Am J Nurs. 2011;110: 47-52.

7. Noteboom JT AS, Cleland JA, Whitman JM. A Primer on Selected Aspects of Evidence-Based Practice Relating to Questions of Treatment, Part 2: Interpreting Results, Application to Clinical Practice, and Self-Evaluation. . journal of orthopaedic \& sports physical therap. 2008;38(8):485.

8. Ubbink DT GG, Vermeulen H. Framework of policy recommendations for implementation of evidencebased practice: A systematic scoping review. BMJ open. 2013;3: e001881.

9. RR. P. Research as evidence: The many meanings. Nurs Sci Q. 2013.

10. Beshir MAB, MPH1; Woreta, Solomon A. BSc, MPH1; Kebede, Mihiretu BSc, MPH1,2 Evidence-based practice among health professionals in hospitals of Northwest Ethiopia. International Journal of Evidence-Based Healthcare. 2017;15 - Issue 4 - p 161-170 doi: 10.1097/XEB.0000000000000111.

11. G. Hadgu SA, S. Tsehay. Assessment of Nurses' Perceptions and Barriers on Evidence Based Practice in Tikur Anbessa Specialized Hospital Addis Ababa Ethiopia. American Journal of Nursing Science. 
2015;Vol. 4, No. 3, 2015, pp. 73-83.

12. Kristensen N, Nymann, C. \& Konradsen, H. Implementing research results in clinical practice- the experiences of healthcare professionals. BMC Health Serv Res. 2015;16, 48.

13. J H. An approach to building research capacity for health practitioners in a public health environment: an organisational perspective. Aust Health Rev. 2014;38(3).

14. Vinoth Gnana Chellaiyan AM, M. Jasmine, and Fasna Liaquathali. Medical research: Perception and barriers to its practice among medical school students of Chennai. journal of education and health promotion. 2019;8: 134.

15. Dereje B HE, Beharu M. Evidence-based Practice Utilization and Associated Factors among Nurses Working in Public Hospitals of Jimma Zone Southwest Ethiopia: A Cross Sectional Study. Adv Pharmacoepidemiol Drug Saf. 2019;7:321. doi: 10.24105/2327-5146.7.321.

16. Clancy CM CK. Evidence-Based Decision making: Global Evidence, Local Decisions. Health Affair 2015.

17. GroupElsevier. WB. A Decade of Development in Sub-Saharan African Science, Technology,Engineering \& Mathematics Research, 2014.

18. Hegde A, Venkataramana, G., Kulkarni, S. B., Bhaskar, N. N., Jacob, J., and Gangadharappa, S. K. Attitudes, experiences, and barriers to research and publishing among dental postgraduate students of Bengaluru city: a cross-sectional study. J Indian Assoc Public Health Dent. 2017;15, 157-161. doi: 10.4103/jiaphd.jiaphd_11_17.

19. Quality AfHRa. Preventive Service task force. Retrieved July 24: 2-11. 2008.

20. Dawit Hoyiso AAaTM. "Evidence based nursing practice and associated factors among nurses working in Jimma zone public hospitals, Southwest Ethiopia."International Journal of Nursing and Midwifery. 2018;10, no. 5 (2018): 47-53.

21. Melnyk BM F-OE, Mays MZ: The Evidence-Based Practice Beliefs and Implementation Scales: Pshychometric Properties of Two New Instruments. . World views Evid Based Nursing. 2008;5(4):208216.

22. Bostrom AM RA, Ehrenberg A, Gustavsson JP, Wallin . Factors associated with evidence-based practice among registered nurses in Sweden: a national cross-sectional study. BMC Health Services Research. 2013;13(1):165.

23. P.Jordan CB, D, . Barriers to implement EBP in a private ICU in the Eastern Cape. South Afrjcrit 2016; care (online) vol.32 n.2 Cape Town.

24. Heydari A ZA. Barriers to and Facilitators of Research Utilization among Iranian Nurses: a Literature Review. Journal of Caring Sciences. 2014; 3(4):265-275. 